\title{
An efficient hybrid method for solving fredholm integral equations using triangular functions
}

\author{
Mohamed A. Ramadan ${ }^{1}$ and Mohamed R. Ali ${ }^{2}$ \\ ${ }^{1}$ Department of Mathematics, Faculty of Science, Menoufia University, Egypt \\ ${ }^{2}$ Department of Mathematics, Faculty of Engineering, Benha University, Egypt
}

Received: 5 March 2016, Accepted: 8 June 2016

Published online: 19 March 2017.

\begin{abstract}
In this paper the orthogonal triangular function (TF) based method is first applied to transform the Fredholm integral equations and Fredholm system of integral equations to a coupled system of matrix algebraic equations. The obtained system is a variant of coupled Sylvester matrix equations. A finite iterative algorithm is then applied to solve this system to obtain the coefficients used to get the form of approximate solution of the unknown functions of the integral problems. Some numerical examples are solved to illustrate the accuracy and the efficiency of the proposed hybrid method. The obtained numerical results are compared with other numerical methods and the exact solutions.
\end{abstract}

Keywords: Fredholm integral equation, triangular functions, generalized Sylvester matrix equation, generalized iterative algorithm.

\section{Introduction}

Many problems in physics, mechanics, economics, sociology and biological lead to the Volterra Integral Equations (VIEs) [1]. These systems are dependent on a noise source, on a Gaussian white noise, so modeling such phenomena naturally requires the use of various Volterra integral equations. The concerned with function spaces spanned by polynomials for which the kernel of the corresponding transforming integral operator is separable being comprised of polynomial functions only, then several approximate methods of solution of integral equations can be developed. A computational approaches to solve integral equations is an essential work in scientific research, for interested reader see to the recent work presented by Y. Suayip [2-7]. Fredholm integral equation is one of the most important integral equations. A computational approach to solving integral equation is an essential work in scientific research. Some methods for solving second kind Fredholm integral equation are available in the open literature. The $B$-spline wavelet method, the method of moments based on $B$-spline wavelets by Maleknejad and Sahlan [8], and variational iteration method (VIM) by He [9-11] have been applied to solve second kind Fredholm linear integral equations. The learned researchers Maleknejad et al. Numerical methods for solving linear Fredholm integral equations system of second kind using Rationalized Haar functions method, Block-Pulse functions, and Taylor series expansion method [12-14]. Haar wavelet method with operational matrices of integration [15] has been applied to solve system of linear Fredholm integral equations of second kind. Quadrature method [16], B-spline wavelet method [17], wavelet Galerkin method [18], and also VIM [19] can be applied to solve nonlinear Fredholm integral equation of second kind. Some iterative methods like Homotopy perturbation method (HPM) [20-21] and Adomian decomposition method (ADM) [22] have been applied to solve nonlinear Fredholm integral equation of second kind.

This paper is organized as follows. First the orthogonal triangular functions (TFs) and their properties are provided in Section 2. In section 3 a finite iterative method is presented to solve couple system of matrix equations. In section 4 the 
suggested hybrid method is presented. The illustrative examples and numerical results obtained via this method are presented in section 5 .

\section{Review of orthogonal triangular functions}

Triangular functions have been introuduced by Deb et al. [23] and studied and used by Babolian et al. [24]. In this section, definitions of vector forms of TFs vector forms and their properties proposed by Babolian et al. [25] are reviewed.

Definition 1.A set of block-pulse functions $(B P F) \psi_{(\mathrm{m})}(t)$ containing $m$ component functions in the semi-open interval $(0$, T) is given by

$$
\psi_{(\mathrm{m})}(t)=\left[\psi_{0}(t) \psi_{1}(t) \ldots \psi_{i}(t) \ldots \psi_{m-1}(t)\right]^{T}
$$

where $[\ldots]^{T}$ denotes transpose.

The ith component $\psi_{i}(\mathrm{t})$ of the BPF vector $\psi_{(\mathrm{m})}(\mathrm{t})$ is defined as

$$
\psi_{i}(t)=\left\{\begin{array}{lc}
1 & (i h) \leq t<(i+1) h \\
0, & \text { elsewhere }
\end{array}\right.
$$

where $i=0,1,2, \cdots,(m-1)$ and $h=\frac{T}{m}$, for more details see [26 ].

A square integrable time function $f(t)$ of Lebesgue measure may be expanded into an m-term BPF series in $\mathrm{t} \in[0, \mathrm{~T})$ as

$$
\mathrm{f}(\mathrm{t}) \approx\left[\begin{array}{lllll}
c_{0} & c_{1} & c_{2} \cdots c_{i} \cdots c_{m-1}
\end{array}\right] \psi_{\mathrm{m}}(t) \cong C^{T} \psi_{\mathrm{m}}(t)
$$

The constant coefficients $c_{i}$ 'sin Eq. (2) are given by

$$
c_{i}=(1 / h) \int_{i h}^{(i+1) h} f(\mathrm{t}) d t
$$

where $h=\frac{T}{m}$ is the duration of each component BPF along time scale.

Definition 2.Let $\psi_{\mathrm{i}}(t)$ be the ith component of an $m$-set of BPFs, we introduce

$$
\psi_{\mathrm{i}}(\mathrm{t})=T 1_{i}(\mathrm{t})+T 2_{i}(\mathrm{t})
$$

Where $T 1_{i}(\mathrm{t})$ and $T 2_{i}(\mathrm{t})$ are the $i$ th components of two m-sets of triangular functions over the interval $[0, T)$ as the following form

$$
\begin{gathered}
\mathrm{T}_{\mathrm{i}}(\mathrm{t})=\left\{\begin{array}{lc}
1-\frac{t-i h}{h} & \text { ih } \leq t<(i+1) h \\
0, & \text { elsewhere }
\end{array}\right. \\
\mathrm{T}_{\mathrm{i}}(\mathrm{t})=\left\{\begin{array}{lc}
\frac{t-i h}{h} & i h \leq t<(i+1) h \\
0, & \text { elsewhere }
\end{array}\right.
\end{gathered}
$$

where $i=0,1,2, \cdots, m-1$, with the number of elementary functions $m$, we consider $h=\frac{T}{m}$ and $\mathrm{T}_{\mathrm{i}}(\mathrm{t})$ as the ith lefthanded triangular function and $\mathrm{T} 2 \mathrm{i}(\mathrm{t})$ as the ith right-handed triangular function. We assumed that $T=1$, so TFs are defined over [0,1) and $h=\frac{1}{m}$. From the definition of TFs, it is clear that TFs are disjoint, orthogonal, and complete [23]. Therefore, we can write

$$
\int_{0}^{1} \mathrm{~T} 1_{\mathrm{i}}(\mathrm{t}) \mathrm{T} 1_{\mathrm{j}}(\mathrm{t})=\int_{0}^{1} \mathrm{~T} 2_{\mathrm{i}}(\mathrm{t}) \mathrm{T} 2_{\mathrm{j}}(\mathrm{t})= \begin{cases}\frac{h}{3}, & i=j \\ 0, & \text { otherwise. }\end{cases}
$$


Let $\mathbf{T}(\mathrm{t})$ be a $2 \mathrm{~m}$-vector defined as

$$
\mathbf{T}(\mathrm{t})=\left(\begin{array}{l}
\mathbf{T} 1(\mathrm{t}) \\
\mathbf{T} 2(\mathrm{t})
\end{array}\right)
$$

The set of TFs may be written as the two vectors $\mathbf{T} 1(\mathrm{t})$ and $\mathbf{T} 2(\mathrm{t})$ as follows

$$
\begin{aligned}
& \mathbf{T} 1(\mathrm{t})=\left[T 1_{0}(\mathrm{t}), \cdots, T 1_{m-1}(\mathrm{t})\right],{ }^{T} \\
& \mathbf{T} 2(\mathrm{t})=\left[T 2_{0}(\mathrm{t}), \cdots, T 2_{m-1}(\mathrm{t})\right],{ }^{T}
\end{aligned}
$$

where $\mathbf{T} 1(\mathrm{t})$ and $\mathbf{T} 2(\mathrm{t})$ are called the left-handed triangular function (LHTF) vector and the right-handed triangular function (RHTF) vector respectively.

\subsection{Multiplication of TFs}

Multiplication of triangular functions and related properties were first treated in [26]. It can be concluded from orthogonality of TFs that

$$
\begin{aligned}
& \mathbf{T 1}(\mathrm{t}) \cdot \mathbf{T} 1^{T}(\mathrm{t})= {\left[\begin{array}{llll}
T 1_{0}(\mathrm{t}) & 0 & \cdots & 0 \\
0 & T 1_{1}(\mathrm{t}) & \cdots & 0 \\
\vdots & \vdots & \ddots & \vdots \\
0 & & T 1_{m-1}(\mathrm{t})
\end{array}\right], } \\
& \mathbf{T 2}(\mathrm{t}) \cdot \mathbf{T 2}^{T}(\mathrm{t})=\left[\begin{array}{llll}
T 2_{0}(\mathrm{t}) & 0 & \cdots & 0 \\
0 & T 2_{1}(\mathrm{t}) & \cdots & 0 \\
\vdots & \vdots & \ddots & \vdots \\
0 & & T 2_{m-1}(\mathrm{t})
\end{array}\right]
\end{aligned}
$$

and

$$
\begin{aligned}
& \mathbf{T} 1(t) \cdot \mathbf{T 2}^{\mathbf{T}}(\mathrm{t}) \cong \mathbf{0} \\
& \mathbf{T} 2(\mathrm{t}) \cdot \mathbf{T} \mathbf{1}^{\mathbf{T}}(\mathrm{t}) \cong \mathbf{0}
\end{aligned}
$$

where $\mathbf{0}$ denotes an $m \times m$ zero matrix.

$$
\begin{aligned}
& \int_{0}^{T} \mathbf{T 1}(\mathrm{t}) \cdot \mathbf{T} 1^{T}(\mathrm{t}) d t=\int_{0}^{T} \mathbf{T} 2(\mathrm{t}) \cdot \mathbf{T} 2(\mathrm{t})^{T}(\mathrm{t}) d t \cong \frac{h}{3} \mathbf{I} \\
& \int_{0}^{T} \mathbf{T} \mathbf{1}(\mathrm{t}) \cdot \mathbf{T} 2^{T}(\mathrm{t}) d t=\int_{0}^{T} \mathbf{T} 2(\mathrm{t}) \cdot \mathbf{T} \mathbf{1}^{T}(\mathrm{t}) d t \cong \frac{h}{6} \mathbf{I}
\end{aligned}
$$

in which $\mathbf{I}$ is $\mathrm{m} \times \mathrm{m}$ identity matrix, for more details see [27]. We propose a numerical method based on TFs to obtain the solution of Fredholm integral equation and the coupled system of Fredholm integral equation.

\subsection{Triangular functions (TFs) for function approximation}

Let $f(\mathrm{t})$ be an $L^{2}[0, \mathrm{~T})$ function, the expansion of any function $\mathrm{f}(\mathrm{t})$ with respect to TFs can be written as follow.

$$
f(t) \cong F_{1}^{T} \mathbf{T} \mathbf{1}(t)+F_{2}^{T} \mathbf{T} \mathbf{2}(t)
$$


where $F_{1}$ and $F_{2}$ are the coefficients of TFs with $F_{1_{i}}=f(i h)$ and $F_{2_{i}}=f((i+1) h)$, for $i=0,1, \cdots, m-1$ so $2 m$ - vector $F$ is defined as,

$$
F=\left(\begin{array}{l}
F 1 \\
F 2
\end{array}\right)
$$

Moreover, for each function $k(t, s)$ is a function of two variables. It can be expanded with respect to TFs as follows $k(t, s) \cong \mathbf{T}^{\mathrm{T}}(\mathrm{t}) \mathrm{K} \mathbf{T}(\mathrm{s})$, where, $\mathbf{T}(\mathrm{t})$ and $\mathbf{T}(\mathrm{s})$ are $2 m_{1}$ - dimensional and $2 m_{2}$-dimensional triangular vectors and $\mathrm{K}$ is a $2 m_{1} \times 2 m_{2}$ coefficient matrix of TFs. For convenience, we put $m_{1}=m_{2}=m$. So, matrix K can be written as

$$
\mathrm{K}=\left(\begin{array}{cc}
(K 11)_{\mathrm{m} \times \mathrm{m}} & (K 12)_{\mathrm{m} \times \mathrm{m}} \\
(K 21)_{\mathrm{m} \times \mathrm{m}} & (K 22)_{\mathrm{m} \times \mathrm{m}}
\end{array}\right)
$$

where $K 11, K 12, K 21$ and $K 22$ are $m \times m$ matrices and can be obtained easily by sampling the function $k(t, s)$ at points $s_{i}$ and $t_{j}$ suchthat $s_{i}=i$ and $t_{j}=j h$, for $i, j=0,1, \cdots, m-1$. Therefore,

$$
\begin{aligned}
& (K 11)_{i j}=k(i h, j h), i=0,1, \cdots, m-1, j=0,1, \cdots, m-1 \\
& (K 12)_{i j}=k(i h,(j+1) h), i=0,1, \cdots, m-1, j=0,1, \cdots, m-1 \\
& (K 21)_{i j}=k((i+1) h, j h), i=0,1, \cdots, m-1, j=0,1, \cdots, m-1 \\
& (K 22)_{i j}=k((i+1) h,(j+1) h), i=0,1, \cdots, m-1, j=0,1, \cdots, m-1 .
\end{aligned}
$$

\section{Solving coupled system of matrix equations using finite iterative algorithm}

We concerned with iterative solutions to coupled system of like forms of the Sylvester matrix equations [26]. There are many variant forms of finite iterative algorithms for solving matrix equation.

$$
A V+B W=C
$$

and coupled system of Sylvester matrix equations

$$
\begin{aligned}
& A_{1} V+B_{1} W=C_{1} \\
& A_{2} V+B_{2} W=C_{2}
\end{aligned}
$$

A finite iterative algorithm is constructed to solve the matrix equation (16) as follows,

\subsection{Algorithm}

1 - input $A, B, C$

2- choose arbitrary matrices $V_{1_{1}} \in \mathfrak{R}^{n \times p}$ and $V_{2_{1}} \in \mathfrak{R}^{r \times p}$

3- set

$$
\begin{aligned}
R_{1} & =C-A V_{1}-B W_{1} \\
P_{1} & =A^{T} R_{1} \\
Q_{1} & =B^{T} R_{1} \\
K & =1
\end{aligned}
$$

4- if $R_{k}=0$ then stop and $V_{k}, W_{k}$ is the solution else let $k=k+1$ go to step 5, 
5- compute

$$
\begin{aligned}
V_{k+1} & =V_{k}+\frac{\left\|R_{k}\right\|^{2}}{\left\|P_{k}\right\|^{2}+\left\|Q_{k}\right\|^{N^{2}}} P_{k}, \quad W_{k+1}=W_{k}+\frac{\left\|R_{k}\right\|^{2}}{\left\|P_{k}\right\|^{2}+\left\|Q_{k}\right\|^{2}} Q_{k} \\
R_{k+1} & =C-A V_{k+1}-B W_{k+1}=R_{k}-\frac{\left\|R_{k}\right\|^{2}}{\left\|P_{k}\right\|^{2}+\left\|Q_{k}\right\|^{2}}\left[A P_{k}+B Q_{k}\right] \\
P_{k+1} & =A^{T} R_{k+1}+\frac{\left\|R_{k+1}\right\|^{2}}{\left\|R_{k}\right\|^{2}} P_{k}, Q_{k+1}=B^{T} R_{k+1}+\frac{\left\|R_{k+1}\right\|^{2}}{\left\|R_{k}\right\|^{2}} Q_{k}
\end{aligned}
$$

This algorithm is a special form of the algorithm considered by M. A Ramadan et al [29].

For the coupled system of Sylvester matrix equations (17), a finite iterative algorithm is presented as follows.

\subsection{Algorithm}

1- input $A_{1}, B_{1}, A_{2}, B_{2}, C_{1}, C_{2}$

2- choose arbitrary matrices $Y_{1_{1}} \in C^{n \times p}$ and $Y_{2_{1}} \in C^{r \times p}$

3- set

$$
\begin{aligned}
& R_{1}=\operatorname{diag}\left(C_{1}-f\left(Y_{1_{1}}, Y_{2_{1}}\right),\left(C_{2}-g\left(Y_{1_{1}}, Y_{2_{1}}\right)\right)\right. \\
& S_{1}=A_{1}{ }^{T}\left(C_{1}-f\left(Y_{1_{1}}, Y_{2_{1}}\right)\right)+A_{2}{ }^{T}\left(C_{2}-g\left(Y_{1_{1}}, Y_{2_{1}}\right)\right) \\
& T_{1}=B_{1}{ }^{T}\left(C_{1}-f\left(Y_{1_{1}}, Y_{2_{1}}\right)\right)+B_{2}{ }^{T}\left(C_{2}-g\left(Y_{1_{1}}, Y_{2_{1}}\right)\right)
\end{aligned}
$$

4- if $R_{k}=0$ then stop and $Y_{1_{k}}, Y_{2_{k}}$ is the solution else let $k=k+1$ go to step 5 .

5 - compute

$$
\begin{aligned}
Y_{1_{k+1}} & =Y_{1 k}+\frac{\left\|R_{k}\right\|^{2}}{\left\|S_{k}\right\|^{2}+\left\|T_{k}\right\|^{2}} S_{k} \\
Y_{2_{k+1}} & =Y_{2 k}+\frac{\left\|R_{k}\right\|^{2}}{\left\|S_{k}\right\|^{2}+\left\|T_{k}\right\|^{2}} T_{k} \\
R_{k+1} & =\operatorname{diag}\left(C_{1}-f\left(Y_{1_{k+1}}, Y_{2_{k+1}}\right),\left(C_{2}-g\left(Y_{1_{k+1}}, Y_{2_{k+1}}\right)\right)\right. \\
& =R_{k}-\frac{\left\|R_{k}\right\|^{2}}{\left\|S_{k}\right\|^{2}+\left\|T_{k}\right\|^{2}} \operatorname{diag}\left(f\left(S_{k}, T_{k}\right), g\left(S_{k}, T_{k}\right)\right) \\
S_{k+1} & =A_{1}^{T}\left(C_{1}-f\left(Y_{1_{k+1}}, Y_{2_{k+1}}\right)\right)+A_{2}^{T}\left(C_{2}-g\left(Y_{1_{k+1}}, Y_{2_{k+1}}\right)\right)+\frac{\left\|R_{k+1}\right\|^{2}}{\left\|R_{k}\right\|^{2}} S_{k} \\
T_{k+1} & =B_{1}{ }^{T}\left(C_{1}-f\left(Y_{1_{k+1}}, Y_{2_{k+1}}\right)\right)+B_{2}^{T}\left(C_{2}-g\left(Y_{1_{k+1}}, Y_{2_{k+1}}\right)\right)+\frac{\left\|R_{k+1}\right\|^{2}}{\left\|R_{k}\right\|^{2}} T_{k}
\end{aligned}
$$

This algorithm is a special form of the algorithm considered by M. A Ramadan et. al. [28].

\section{Problem statement}

In this section, we present a hybrid method. The suggested technique is first applying TF method to transform the Fredholm integral equations to a coupled system of matrix algebraic equations. The obtained system is a variant of 
coupled Sylvester matrix equations then we apply algorithm 3.2 to solve the resultant coupled system of Sylvester matrix equations to compute our solution function for the original problem. First, consider the following equation.

$$
y(\mathrm{t})=f(t)+\lambda \int_{0}^{1} k(\mathrm{t}, \mathrm{s}) y(\mathrm{~s}) d s
$$

where $f(\mathrm{t}) \in L^{2}([0,1)), k(\mathrm{t}, \mathrm{s}) \in L^{2}([0,1)) \times L^{2}([0,1))$ and $y(\mathrm{t})$ is the unknown function. This problem is to determine TF pair coefficients of $y(\mathrm{t})$ in the interval $[0,1)$; from the known functions $f(\mathrm{t})$ and kernel $k(\mathrm{t}, \mathrm{s})$. We expand $f(\mathrm{t})$ and $y(\mathrm{t})$ by TFs (LHTF and RHTF) as follows,

$$
\begin{aligned}
& f(t) \cong F_{1}^{T} \mathbf{T} \mathbf{1}(\mathrm{t})+F_{2}^{T} \mathbf{T} 2(\mathrm{t}) \\
& y(t) \cong Y_{1}^{T} \mathbf{T} \mathbf{1}(\mathrm{t})+Y_{2}^{T} \mathbf{T} 2(\mathrm{t})
\end{aligned}
$$

We can expand $k(t, s)$ in the interval $[0,1)$ by TFs. Suppose that this approximation be as follows,

$$
k(t, s)=\mathbf{T 1}(t)^{T}(t) \cdot K 11 \cdot \mathbf{T} 1(s)+\mathbf{T 1}^{T}(t) \cdot K 12 \cdot \mathbf{T} 2(s)+\mathbf{T 2}^{T}(t) \cdot K 21 \cdot \mathbf{T} \mathbf{1}(s)+\mathbf{T 2}^{T}(t) \cdot K 22 \cdot \mathbf{T} 2(s)
$$

where $K 11, K 12, K 21$ and $K 22$ are obtained from Eqs. (15). Then, we have

$$
\begin{aligned}
Y_{1}{ }^{T} \mathbf{T 1}(\mathrm{t})+Y_{2}{ }^{T} \mathbf{T} 2(\mathrm{t}) & =F_{1}{ }^{T} \mathbf{T} \mathbf{1}(\mathrm{t})+{F_{2}}^{T} \mathbf{T} \mathbf{2}(\mathrm{t})+\lambda \int_{0}^{1}\left[\left(\mathbf{T 1}^{T}(\mathrm{t}) K 11 \mathbf{T} \mathbf{1}(\mathrm{s})+\mathbf{T 1}^{T}(\mathrm{t}) K 12 \mathbf{T} 2(\mathrm{~s})\right.\right. \\
& \left.\left.+\mathbf{T 2}^{T}(\mathrm{t}) K 21 \mathbf{T} \mathbf{1}(\mathrm{s})+\mathbf{T 2}^{T}(\mathrm{t}) K 22 \mathbf{T} \mathbf{2}(\mathrm{s})\right)\left(Y_{1}{ }^{T} \mathbf{T} \mathbf{1}(s)+Y_{2}{ }^{T} \mathbf{T} 2(\mathrm{~s})\right) d s\right]
\end{aligned}
$$

We have

$$
\begin{aligned}
& Y_{1}{ }^{T} \mathbf{T} \mathbf{1}(\mathrm{t})+Y_{2}{ }^{T} \mathbf{T} \mathbf{2}(\mathrm{t})=F_{1}{ }^{T} \mathbf{T} 1(\mathrm{t})+F_{2}{ }^{T} \mathbf{T} \mathbf{2}(\mathrm{t})+\lambda\left[K 11^{T} \mathbf{T} \mathbf{1}(\mathrm{t}) Y_{1}{ }^{T} \int_{0}^{1} \mathbf{T} \mathbf{1}(\mathrm{s}) \mathbf{T} \mathbf{1}^{T}(\mathrm{~s}) d s\right. \\
& +K 12^{T} \mathbf{T} \mathbf{1}(\mathrm{t}) Y_{1}{ }^{T} \int_{0}^{1} \mathbf{T 1}(\mathrm{s}) \mathbf{T 2}^{T}(\mathrm{~s}) d s+K 21^{T} \mathbf{T} \mathbf{2}(\mathrm{t}) Y_{1}^{T} \int_{0}^{1} \mathbf{T} 1(\mathrm{~s}) \mathbf{T} \mathbf{1}^{T}(\mathrm{~s}) d s \\
& +K 22^{T} \mathbf{T} 2(\mathrm{t}) Y_{1}{ }^{T} \int_{0}^{1} \mathbf{T 1}(\mathrm{s}) \mathbf{T 2}^{T}(\mathrm{~s}) d s+K 11^{T} \mathbf{T} \mathbf{1}(\mathrm{t}) Y_{2}{ }^{T} \int_{0}^{1} \mathbf{T} 2(\mathrm{~s}) \mathbf{T 1}^{T}(\mathrm{~s}) d s \\
& +K 12^{T} \mathbf{T 1}(\mathrm{t}) Y_{2}{ }^{T} \int_{0}^{1} \mathbf{T} \mathbf{2}(\mathrm{s}) \mathbf{T} 2^{T}(\mathrm{~s}) d s+K 21^{T} \mathbf{T} 2(\mathrm{t}) Y_{2}{ }^{T} \int_{0}^{1} \mathbf{T} 2(\mathrm{~s}) \mathbf{T} 1^{T}(\mathrm{~s}) d s \\
& \left.+K 22^{T} \mathbf{T} 2(\mathrm{t}) Y_{2}^{T} \int_{0}^{1} \mathbf{T} 2(\mathrm{~s}) \mathbf{T}^{T} d s\right]
\end{aligned}
$$

Then by using Eqs. (11) we have

$$
\begin{aligned}
Y_{1}{ }^{T} \mathbf{T} 1(\mathrm{t})+ & Y_{2}{ }^{T} \mathbf{T} 2(\mathrm{t})=F_{1}{ }^{T} \mathbf{T} 1(\mathrm{t})+F_{2}{ }^{T} \mathbf{T} 2(\mathrm{t}) \\
& +\lambda\left[Y_{1}{ }^{T}\left(\frac{h}{3} K 11^{T} \mathbf{T} 1(\mathrm{t})+\frac{h}{6} K 12^{T} \mathbf{T} 1(\mathrm{t})+\frac{h}{3} K 21^{T} \mathbf{T} 2(\mathrm{t})+\frac{h}{6} K 22^{T} \mathbf{T} \mathbf{2}(\mathrm{t})\right)\right. \\
& \left.+Y_{2}{ }^{T}\left(\frac{h}{6} K 11^{T} \mathbf{T} \mathbf{1}(\mathrm{t})+\frac{h}{3} K 12^{T} \mathbf{T} \mathbf{T}(\mathrm{t})+\frac{h}{6} K 21^{T} \mathbf{T} \mathbf{2}(\mathrm{t})+\frac{h}{3} K 22^{T} \mathbf{T} 2(\mathrm{t})\right)\right]
\end{aligned}
$$

The coefficients of $\mathbf{T 1}(\mathrm{t})$ and $\mathbf{T} 2(\mathrm{t})$ on both sides of the above equation must be equal; hence, we have the following equations for the corresponding coefficients of TFs,

$$
\begin{aligned}
Y_{1}{ }^{T}\left(I-\lambda\left(\frac{h}{3} K 11^{T}+\frac{h}{6} K 12^{T}\right)\right)-\lambda Y_{2}{ }^{T}\left(\frac{h}{6} K 11^{T}+\frac{h}{3} K 12^{T}\right) & =F 1^{T} \\
\left.-\lambda Y_{1}{ }^{T}\left(\frac{h}{3} K 21^{T}+\frac{h}{6} K 22^{T}\right)\right)+Y_{2}{ }^{T}\left(I-\lambda\left(\frac{h}{6} K 21^{T}+\frac{h}{3} K 22^{T}\right)\right) & =F 2^{T}
\end{aligned}
$$


set

$$
\begin{aligned}
& A_{1}=\left(I-\lambda\left(\frac{h}{3} K 11^{T}+\frac{h}{6} K 12^{T}\right)\right) \\
& A_{2}=-\lambda Y_{2}^{T}\left(\frac{h}{6} K 11^{T}+\frac{h}{3} K 12^{T}\right) \\
& \left.B_{1}=-\lambda Y_{1}^{T}\left(\frac{h}{3} K 21^{T}+\frac{h}{6} K 22^{T}\right)\right) \\
& B_{2}=\left(I-\lambda\left(\frac{h}{6} K 21^{T}+\frac{h}{3} K 22^{T}\right)\right)
\end{aligned}
$$

Then, we have the following linear system:

$$
\begin{aligned}
& A_{1} Y_{1}+B_{1} Y_{2}=F_{1} \\
& A_{2} Y_{1}+B_{2} Y_{2}=F_{2} .
\end{aligned}
$$

Now, let $f\left(Y_{1}, Y_{2}\right)=A_{1} Y_{1}+B_{1} Y_{2}$ and $g\left(Y_{1}, Y_{2}\right)=A_{2} Y_{1}+B_{2} Y_{2}$

For the above linear system we can find $Y_{1}$ and $Y_{2}$ using the suggested efficient finite iterative algorithm 3.2.

In this section a generalization of the method introduced in the above is presented to tackle the coupled system of Fredholm integral equation [30].

$$
y_{i}(\mathrm{t})=f_{i}(\mathrm{t})+\lambda \sum_{i=1}^{n} \int_{0}^{1} k_{i j}(\mathrm{t}, \mathrm{s}) y_{j}(\mathrm{~s}) d s, \quad i=1 \ldots, n,
$$

where $f_{i}(\mathrm{t}) \in L^{2}([0,1))$, and the kernels $k_{i j}(\mathrm{t}, \mathrm{s}) \in L^{2}([0,1)) \times L^{2}([0,1))$ are known $, i, j=1,2, \ldots, n$. and $y_{i}(\mathrm{t})$ are the unknown functions. This problem is to determine TF pair coefficients of $y_{i}(\mathrm{t})$ in the interval $[0,1)$; from the known functions $f_{i}(\mathrm{t})$ and kernel $k_{i j}(\mathrm{t}, \mathrm{s})$. We expand $f(\mathrm{t})$ and $y(\mathrm{t})$ by TFs (LHTF and RHTF) as follows,

$$
\begin{aligned}
& f_{i}(\mathrm{t}) \cong F_{1_{i}}^{T} \mathbf{T} \mathbf{1}(\mathrm{t})+F_{2_{i}}^{T} \mathbf{T} \mathbf{2}(\mathrm{t}) \\
& y_{i}(\mathrm{t}) \cong Y_{1_{i}}^{T} \mathbf{T} \mathbf{1}(\mathrm{t})+Y_{2_{i}}^{T} \mathbf{T} 2(\mathrm{t})
\end{aligned}
$$

By using Eqs. (11) and (20), we approximate the kernel $k_{i j}(t, s)$ by TFs as

$$
\begin{aligned}
\int_{0}^{1} k_{i j}(t, s) y_{j}(s) d s & =\left[Y _ { 1 _ { j } } { } ^ { T } \left(\frac{h}{3} K 11_{i j}{ }^{T} \mathbf{T} \mathbf{1}(\mathrm{t})+\frac{h}{6} K 12_{i j}{ }^{T} \mathbf{T} \mathbf{1}(\mathrm{t})+\frac{h}{3} K 11_{i j}{ }^{T} \mathbf{T} 2(\mathrm{t})\right.\right. \\
& \left.+\frac{h}{6} K 22_{i j}{ }^{T} \mathbf{T} 2(\mathrm{t})\right)+Y_{2_{j}}{ }^{T}\left(\frac{h}{6} K 11_{i j}{ }^{T} \mathbf{T} \mathbf{1}(\mathrm{t})+\frac{h}{3} K 12_{i j}{ }^{T} \mathbf{T} \mathbf{1}(\mathrm{t})\right. \\
& \left.\left.+\frac{h}{6} K 21_{i j}{ }^{T} \mathbf{T} 2(\mathrm{t})+\frac{h}{3} K 22_{i j}{ }^{T} \mathbf{T} 2(\mathrm{t})\right)\right]
\end{aligned}
$$

Substituting the Eqs. (24) and (25) into Eq. (23) and equating the like coefficients of TFs, we get the following system.

$$
\begin{aligned}
& \sum_{j=1}^{n}\left[Y_{1}^{T}\left(\Delta_{i j}-\lambda\left(\frac{h}{3} K 11_{i j}{ }^{T}+\frac{h}{6} K 12_{i j}{ }^{T}\right)\right)-\lambda Y_{2}{ }^{T}\left(\frac{h}{6} K 11_{i j}{ }^{T}+\frac{h}{3} K 12_{i j}{ }^{T}\right)\right]=F 1_{i}{ }^{T} \\
& \left.\sum_{j=1}^{n}\left[-\lambda Y_{1}{ }^{T}\left(\frac{h}{3} K 21_{i j}{ }^{T}+\frac{h}{6} K 22_{i j}{ }^{T}\right)\right)+Y_{2}{ }^{T}\left(\Delta_{i j}-\lambda\left(\frac{h}{6} K 21_{i j}{ }^{T}+\frac{h}{3} K 22_{i j}{ }^{T}\right)\right)\right]=F 2_{i}{ }^{T}
\end{aligned}
$$


set

$$
\begin{aligned}
& A 1_{i j}=\left(\Delta_{i j}-\lambda\left(\frac{h}{3} K 11_{i j}{ }^{T}+\frac{h}{6} K 12_{i j}{ }^{T}\right)\right) \\
& \left.B 1_{i j}=-\lambda Y_{1}^{T}\left(\frac{h}{3} K 21_{i j}{ }^{T}+\frac{h}{6} K 22_{i j}{ }^{T}\right)\right) \\
& B 2 i j=\left(\Delta_{i j}-\lambda\left(\frac{h}{6} K 21_{i j}{ }^{T}+\frac{h}{3} K 22_{i j}{ }^{T}\right)\right)
\end{aligned}
$$

and

$$
\Delta_{i j}= \begin{cases}\mathbf{I}, & i=j \\ 0 & i \neq j\end{cases}
$$

for $i, j=1,2, \ldots n$ and $\mathbf{I}$ is an identity matrix. Then, we have the following linear system,

$$
\begin{aligned}
& A_{1 i j} Y_{1}+B_{1_{i j}} Y_{2}=F 1_{i} \\
& A_{2_{i j}} Y_{1}+B 2_{2_{i j}} Y_{2}=F 2_{i} .
\end{aligned}
$$

For the above system we can find $Y_{1}$ and $Y_{2}$ using the suggested finite iterative algorithm 3.2.

\section{Illustrative numerical examples}

In this section, we represent some examples and their numerical results to show the high accuracy of the solution obtained by TFs and then we compare all results with the exact solution.

Example 1. Consider the Fredholm integral equation of the second kind

$$
y(t)=e^{t}-1+\int_{0}^{1} s y(s) d s
$$

where $f(\mathrm{t})=e^{t}-1, k(\mathrm{t}, \mathrm{s})=s$ and the exact solution isy $(\mathrm{t})=e^{t}$. by using TF method, the problem can be solved, for $m=4$ and 32 are listed in tables 1 and 2 clearly compares estimation of the solution obtained via TF method by the direct method using maple and a finite iterative algorithm. We note that the iterative method is obtained the same results as the inverse with the direct method as shown in Table 1.

Table 1: Numerical results obtained for $m=4$ in Example 1 via TF method by using a finite iterative algorithm.

\begin{tabular}{|c|c|c|c|c|}
\hline$t$ & \multicolumn{2}{|c|}{ Direct method } & \multicolumn{2}{c|}{ Iterative method } \\
\hline & $Y_{1}$ & $Y_{2}$ & $Y_{1}$ & $Y_{2}$ \\
\hline 0 & 1.01033142 & 1.29435683 & 1.01033142 & 1.29435683 \\
\hline 0.25 & 1.29435683 & 1.65905269 & 1.29435683 & 1.65905269 \\
\hline 0.5 & 1.65905269 & 2.12733143 & 1.65905269 & 2.12733143 \\
\hline 0.75 & 2.12733143 & 2.72861324 & 2.12733143 & 2.72861324 \\
\hline
\end{tabular}


Table 2: Numerical results obtained for $m=32$ in Example 1 via TF method by using a finite iterative algorithm.

\begin{tabular}{|c|c|c|}
\hline $\mathrm{t}$ & TF method & Exact solution \\
\hline 0 & 0.999844 & 1 \\
\hline 0.1 & 1.105420 & 1.105171 \\
\hline 0.2 & 1.492294 & 1.2214027 \\
\hline 0.3 & 1.349645 & 1.3498588 \\
\hline 0.4 & 1.492103 & 1.4918246 \\
\hline 0.5 & 1.648884 & 1.6487212 \\
\hline 0.6 & 1.822071 & 1.8221188 \\
\hline 0.7 & 2.013375 & 2.0137527 \\
\hline 0.8 & 2.225963 & 2.2255409 \\
\hline 0.9 & 2.459956 & 2.4596031 \\
\hline 1 & 2.718444 & 2.7182818 \\
\hline
\end{tabular}

Example 2. Consider the Fredholm integral equation of the first kind

$$
\frac{\left(e^{x+1}-1\right)}{(x+1)}=\int_{0}^{1} e^{x y} f(y) d x
$$

where $k(x, y)=e^{x y}$ and the exact solution is $f(x)=e^{x}$. By using TF method, the problem can be solved, for $m=32$ and $m=64$ are listed in Tables 3 . We compare estimation of the solution obtained via TF method by the finite iterative algorithm. By increasing $\mathrm{m}$, the computed results have appropriate accuracy and the error of the solution decreases.

Table 3: Numerical results obtained for $m=32$ and 64 in Example 2 via TF method by using a finite iterative algorithm.

\begin{tabular}{|c|c|c|c|}
\hline $\mathrm{t}$ & $\mathrm{M}=32$ & $\mathrm{~m}=64$ & Exact \\
\hline 0 & 1.0072935427 & 1.001519304 & 1 \\
\hline 0.1 & 1.105610016 & 1.105063274 & 1.105171 \\
\hline 0.2 & 1.215828568 & 1.221760050 & 1.2214027 \\
\hline 0.3 & 1.339368626 & 1.344218523 & 1.3498588 \\
\hline 0.4 & 1.477424094 & 1.494374050 & 1.4918246 \\
\hline 0.5 & 1.630883490 & 1.644547770 & 1.6487212 \\
\hline 0.6 & 1.800235075 & 1.819785795 & 1.8221188 \\
\hline 0.7 & 2.027848210 & 2.013982342 & 2.0137527 \\
\hline 0.8 & 2.231443992 & 2.228538315 & 2.2255409 \\
\hline 0.9 & 2.400063129 & 2.451819326 & 2.4596031 \\
\hline 1 & 2.700265588 & 2.718717609 & 2.7182818 \\
\hline
\end{tabular}

The numerical results are shown in table 1, 2 and table 3 . For examples 1 and 2 we obtained our solution $\left(Y_{1}, Y_{2}\right)$ after seven iterations.

Example 3. Consider the following system of linear integral equations of the second kind

$$
\begin{aligned}
& \mathrm{u}_{1}(\mathrm{x})=\frac{3}{4}+\int_{0}^{1} x u_{1}(t) d s+\int_{0}^{1}(x-t) u_{2}(t) d t \\
& \mathrm{u}_{2}(\mathrm{x})=-\frac{1}{12}-x+3 x^{2}+\int_{0}^{1}(x-t) u_{1}(t) d s+\int_{0}^{1} t u_{2}(t) d t
\end{aligned}
$$


with the exact solution $u_{1}(x)=2 x$ and $u_{2}(x)=3 x^{2}$.

By using TF method, the problem is solved, for $\mathrm{m}=256$ and the obtained solutions are listed at different value of $\mathrm{x}$ in Tables 4. We compare estimation of the solution obtained via TF method by the finite iterative algorithm. We obtained our solution $\left(Y_{1}, Y_{2}\right)$ after five iterations.

Table 4: Numerical results obtained for $m=256$ in Example 3 via TF method by using a finite iterative algorithm.

\begin{tabular}{|c|c|c|c|c|}
\hline $\mathrm{x}$ & \multicolumn{2}{|c|}{ TF method } & \multicolumn{2}{c|}{ Exact value of } \\
\hline & $\mathrm{u}_{1}(\mathrm{x})$ & $\mathrm{u}_{2}(\mathrm{x})$ & $\mathrm{u}_{1}(\mathrm{x})$ & $\mathrm{u}_{2}(\mathrm{x})$ \\
\hline 0 & 0.00000 & 0.00000 & 0 & 0 \\
\hline 0.1 & 0.19999 & 0.03001 & 0.2 & 0.03 \\
\hline 0.2 & 0.399996 & 0.12001 & 0.4 & 0.12 \\
\hline 0.3 & 0.599997 & 0.26999 & 0.6 & 0.27 \\
\hline 0.4 & 0.799999 & 0.47999 & 0.8 & 0.48 \\
\hline 0.5 & 1.000000 & 0.74999 & 1 & 0.75 \\
\hline 0.6 & 1.200001 & 1.07999 & 1.2 & 1.08 \\
\hline 0.7 & 1.400002 & 1.46999 & 1.4 & 1.47 \\
\hline 0.8 & 1.600003 & 1.91999 & 1.6 & 1.92 \\
\hline 0.9 & 1.800005 & 2.42999 & 1.8 & 2.43 \\
\hline 1 & 2.000004 & 3.00000 & 2 & 3 \\
\hline
\end{tabular}

\section{Conclusion}

In this article we present a new technique for solving VIEs numerically. Here, a hybrid method of triangular functions and an iterative method are considered. The benefits of this method are lower cost of setting up the system of equations without any integration and to recover the singularity, moreover, the computational cost of operations is low. These advantages make the method easier to apply. It follows from the numerical results that the accuracy of the solutions obtained using the TFs is quite good. The structural properties of TFs are utilized to reduce the Fredholm integral equations to an algebraic equation. It seems that present method is appropriate for linear integral equations system. We test the proposed algorithm using Maple and the results verify our theoretical findings. The numerical results have demonstrated the superiority and efficiency of the proposed method where our method exhibits fast convergence behavior.

\section{Competing interests}

The authors declare that they have no competing interests.

\section{Authors' contributions}

All authors have contributed to all parts of the article. All authors read and approved the final manuscript.

\section{References}

[1] A.M.Wazwaz, Linear and Nonlinear Integral Equations: Methods and Applications, $\quad$ Springer, New York, NY, USA (2011).

[2] Suayip Y, "A collocation method based on Bernstein polynomials to solve nonlinear Fredholm- Volterra integro-differential equations," Applied Mathematics and Computation, Vol.273, No. 2, pp. 142-154 (2016).

[3] Suayip Y, "Numerical solutions of system of linear Fredholm-Volterra integro-differential equations by the Bessel collocation method and error estimation," Applied Mathematics and Computation, Vol.250, No. 1, pp. 320-338 (2015). 
[4] Suayip Y "Laguerre approach for solving pantograph-type Volterra integro-differential equations," Applied Mathematics and Computation, vol.232, pp. 1183-1199 (2014).

[5] Suayip Y, “A Numerical Approximation For Volterra'S Population Growth Model With Fractional Order," Applied Mathematical Modelling, Vol.37, No. 2, pp. 3216-3227 (2013).

[6] Suayip Y, "Numerical Solutions Of Integro-Differential Equations And Application Of A Population Model With An Improved Legendre Method,” Applied Mathematical Modelling, Vol.37, pp. 3216-3227 (2013).

[7] Suayip Ys Niyazi Ş, Mehmet S "Numerical Solutions Of Systems Of Linear Fredholm Integro-Differential Equations With Bessel Polynomial Bases", Computers and Mathematics with Applications, Vol.61, pp.3079-3096 (2011).

[8] K. Maleknejad and M. N. Sahlan, "The method of moments for solution of second kind Fredholm integral equations based on B-spline wavelets," International Journal of Computer Mathematics, Vol. 87, No. 7, pp. 1602-1616 (2010).

[9] J. H. He, "Variational iteration method-a kind of non-linear analytical technique" International Journal of Non-Linear Mechanics, Vol. 34, No. 4, pp. 699-708 (1999).

[10] J. H. He, "Some asymptotic methods for strongly nonlinear equations," International Journal of Modern Physics B, Vol. 20, No. 10, pp. 1141-1199 (2006).

[11] J. H. He, "Variational iteration method—some recent results and new interpretations," Journal of Computational and Applied Mathematics, Vol. 207, No. 1, pp. 3-17 (2007).

[12] K. Maleknejad, M. Shahrezaee, and H. Khatami, "Numerical solution of integral equations system of the second kind by blockpulse functions," Applied Mathematics and Computation, Vol. 166, No. 1, pp. 15-24 (2005).

[13] K. Maleknejad, N. Aghazadeh, and M. Rabbani, "Numerical solution of second kind Fredholm integral equations system by using a Taylor-series expansion method," Applied Mathematics and Computation, Vol. 175, No. 2, pp. 1229-1234 (2006).

[14] K. Maleknejad and F. Mirzaee, "Numerical solution of linear Fredholm integral equations system by rationalized Haar functions method," International Journal of Computer Mathematics, Vol. 80, No. 11, pp. 1397-1405 (2003).

[15] X.Y. Lin, J.-S. Leng, and Y.-J. Lu, "A Haar wavelet solution to Fredholm equations," in Proceedings of the International Conference on Computational Intelligence and Software $\quad$ Engineering (CiSE '09), pp. 1-4 (2009).

[16] M. J. Emamzadeh and M. T. Kajani, "Nonlinear Fredholm integral equation of the second kind with quadrature methods," Journal of Mathematical Extension, Vol. 4, No. 2, pp. 51-58 (2010).

[17] M. Lakestani, M. Razzaghi, and M. Dehghan, "Solution of nonlinear Fredholm- Hammerstein integral equations by using semiorthogonal spline wavelets," Mathematical Problems in Engineering, Vol. 2, No. 1, pp. 113-121 (2005).

[18] Y. Mahmoudi, "Wavelet Galerkin method for numerical solution of nonlinear integral equation," Applied Mathematics and Computation, vol. 167, no. 2, pp. 1119-1129 (2005).

[19] J. Biazar and H. Ebrahimi, "Iterationmethod for Fredholm integral equations of second kind," Iranian Journal of Optimization, Vol. 1, pp. 13-23 (2009).

[20] D. D. Ganji, G. A. Afrouzi, H. Hosseinzadeh, and R. A. Talarposhti, "Application of homotopy-perturbation method to the second kind of nonlinear integral equations," Physics $\quad$ Letters A, Vol. 371, No. 1-2, pp. 20-25 (2007).

[21] M. Javidi and A. Golbabai, "Modified homotopy perturbation method for solving non-linear Fredholm integral equations," Chaos, Solitons and Fractals, Vol. 40, No. 3, pp. 1408-1412 (2009).

[22] E. Babolian, J. Biazar, and A. R. Vahidi, "The decomposition method applied to systems of Fredholm integral equations of the second kind," Applied Mathematics and Computation,

Vol. 148, No. 2, pp. 443-452 (2004).

[23] A. Deb, A. Dasgupta and G. Sarkar, "A new set of orthogonal functions and its application to the analysis of dynamic systems," Journal of The Franklin Institute, Vol. 343, No. 1, pp. 1-26 (2006).

[24] E. Babolian, R. Mokhtari, M. Salmani, "Using direct method for solving variational problems via triangular orthogonal functions," Applied Mathematics and Computation, Vol. 191, No. 2, pp. 206-217 (2007).

[25] E. Babolian, Z. Masouri, S. Hatamzadeh-Varmazyar, "Numerical solution of nonlinear Volterra-Fredholm integro-di_erential equations via direct method using triangular functions, Computers and Mathematics with Applications," Vol. 58, pp. 239-247, (2009).

[26] A. Deb, G. Sarkar, A. Sengupta, "Triangular orthogonal functions for the analysis of continuous time systems," New Delhi: Elsevier, (2007).

[27] E. Babolian, HR. Marzban and M. Salmani, "Using triangular orthogonal functions for solving Fredholm integral equations of the second kind,"Applied Mathematics and Computation, Vol. 201, No. 2, pp. 452-464 (2008).

[28] M. A. Ramadan, T. S. El-Danaf and A. M. E. Bayoumi "A finite iterative algorithm for the solution of Sylvester-conjugate matrix equation $A V+B W=E \bar{V} F+C$ and $\quad A V+B \bar{W}=E \bar{V} F+C$,"Journal of Mathematical and Computer Modelling, Vol. 58, No 11, pp 1738-1754, ( 2013). 
[29] M. A. Ramadan and A. M. E. Bayoumi, "Explicit and iterative methods for solving the matrix equation $A V+B W=E V F+C$," Asian Journal of Control, Vol. 16, No. 2, pp. 965-974 (2014).

[30] K. Maleknejad , M. Shahrezaee , H. Khatami , "Numerical solution of integral equations system block-pulse functions," Applied Mathematics and Computation, Vol. $\quad$ 166, No. 1, pp. 15-24 (2005).

of the second kind by 